\title{
In Situ Measurements of HCN in a Tube Furnace with Infrared Polarization Spectroscopy
}

\author{
ZHIWEI SUN ${ }^{1}$, MICHAEL FÖRSTH ${ }^{2}$, ZHONGSHAN LI $^{1}$, BO LI ${ }^{1}$, and MARCUS ALDÉN ${ }^{1}$ \\ ${ }^{1}$ Division of Combustion Physics \\ Lund University \\ P.O. Box 118, S-221 00 Lund, Sweden \\ ${ }^{2} \mathrm{SP}$ Technical Research Institute of Sweden \\ P.O. Box 857, S-501 15 Borås, Sweden
}

\begin{abstract}
Spatially and temporally resolved in situ measurements of HCN in a steady state tube furnace have been performed using mid-infrared polarization spectroscopy (IRPS). Such measurements are important for the understanding of the detailed HCN chemistry, including pyrolysis, gas phase production, and gas phase consumption. The measurements presented here are in agreement with existing models for HCN chemistry. In particular, it was found that high temperatures and good ventilation result in relatively high peak concentrations of HCN inside the tube furnace. At the same time these conditions also result in high consumption rates of $\mathrm{HCN}$ in the hot fire effluents. The results indicate that, although the peak concentrations are highest for high temperatures and good ventilation conditions, the concentration at the exit of the tube are lower for these conditions than those at lower temperatures and under-ventilated conditions.
\end{abstract}

KEYWORDS: spectroscopy, toxicity, fire chemistry, smoke, HCN, steady state tube furnace.

\section{INTRODUCTION}

At elevated temperatures, such as in fire situations, the smoke chemistry is important near the fire implying that the concentration of important toxic gases, e.g. HCN [1-3], will differ between the sampling location and the measurement chamber. If the detailed chemistry of the production and destruction of specific species is to be studied, it is necessary to have a detailed understanding of the exact composition of species in space as well as in time.

$\mathrm{HCN}$ is a very toxic gas. It is approximately 20 times more toxic than $\mathrm{CO}$ [2] and its intoxication pattern is more rapid [4]. HCN is produced from the pyrolysis of organic nitrogen fuels [2] but can also be formed as an intermediate from combustion of nitrogen free fuels at sufficient temperatures [5]. Under typical fire conditions HCN can be consumed by isomerization to HNC, or by oxidation reactions with radicals, mainly $\mathrm{O}$ and $\mathrm{OH}$ [2]. Investigations using the steady state tube furnace [6,7] using nylon 6,6 show that for well ventilated combustion most $\mathrm{HCN}$ ends up as $\mathrm{NO}_{\mathrm{x}}$ [8], with $\mathrm{NO}_{2}$ as the most important toxicant, whereas for equivalence ratios equal to or greater than one HCN is the most important toxicant produced [9].

Typical experiments in the steady state tube furnace give total yield information for different gases, for different fuels, and for different operating conditions once the fire effluents have left the furnace $[6,7,10$ 12]. However, in order to understand the kinetics behind the total yield of a toxicant, including pyrolysis as well as both production and consumption in the gas phase, in situ measurements are necessary. Such measurements provide information about the dynamics of the toxicant concentration, e.g. HCN, as the gases from the fire plume zone mix with the downstream air inside the furnace.

In the case of gas sampling techniques such as Fourier transform infrared spectroscopy (FTIR) the measurements are conducted in a chamber that is physically separated from the sampling point, at least by the length of the probe. This means that reactive molecules can react or stick to the probe on their way to the measurement chamber, e.g. this is a well known problem with acid gases [4]. In particular, the extensive filtration required when using FTIR spectroscopy, due to the need to remove all soot from the sample prior to entry into the measurement cell, can result in sample loss or change [13].

Using laser diagnostics it is possible to study the chemistry in situ, without gas sampling. Thus, laser diagnostics and chemical modeling are often the combination of choice in combustion sciences [14]. Various laser techniques have been developed and applied to the measurement of temperature, species 
concentration and velocities etc. in recent years. These techniques have been important in the diagnostics of various phenomena in combustion [15]. Several techniques have shown outstanding features, e.g., nonintrusiveness in combination with high temporal ( 10 ns) and spatial resolution ( 50-100 $\mu \mathrm{m})$.

In this work, IRPS (infrared polarization spectroscopy) was used to measure the concentration of HCN in a tube furnace fueled with nylon 6,6 pellets at different ventilation conditions, different temperatures and different positions along the furnace. Polarization spectroscopy (PS) is a widely used nonlinear subDoppler spectroscopic technique [16], which was firstly reported by Wieman and Hänsch [17]. In a representative set-up of PS, a co-propagating strong pump and a weak probe beam, usually derived from the same laser, are tuned to optical transitions of the species studied and crossed at the measurement point. The pump beam induces bi-refringence by polarized optical pumping. The bi-refringence is measured with the probe beam by measuring the polarization rotation. As for most coherent techniques the PS-signal propagates as a laser-like beam. This is an advantage when discriminating the signal from background noise due to non-coherent scattering and fluorescence.

Most PS studies heretofore have been limited to the ultraviolet/visible spectral region by probing electronic transitions. Probing the molecular ro-vibrational transition with infrared (IR) excitation has always been attractive to the combustion diagnostic community. Many important combustion species which are 'dark' in the ultraviolet/visible part of the spectrum, as they post no conveniently accessible electronic transitions, are detectable in the mid-IR spectral range. Due to limited availability of proper IR laser sources, low sensitivity of the infrared detectors and the relatively low fluorescence quantum yields, only limited laserbased combustion diagnostic experiments in the mid-infrared spectral region via ro-vibrational transitions have been reported. Infrared polarization spectroscopy (IRPS) is an absorption-based, crossed-beam, coherent technique with the potential to achieve high sensitivity and high contrast against a thermal background. As such it has proved to be a proper technique to probe molecular ro-vibrational transitions in the mid-infrared spectral range. In the last five years, IRPS has been applied in combustion diagnostics to detect $\mathrm{CH}_{4}$ [18,19], $\mathrm{C}_{2} \mathrm{H}_{6}$ [19], $\mathrm{CO}_{2}$ [20,21], $\mathrm{H}_{2} \mathrm{O}$ [20], $\mathrm{C}_{2} \mathrm{H}_{2}$ [22], $\mathrm{OH}$ [23], and $\mathrm{HCl}$ [24,25].

An advantage of PS compared with other non-linear techniques, e.g. DFWM (degenerate four-wave mixing) and CARS (coherent anti-stokes Raman spectroscopy), is the relatively simple experimental setup. This is represented by the automatic phase matching with crossing of only two beams [26] and the overlap of the signal beam with the probe beam which can be used to guide the signal detection by slightly opening the analyzing polarizer. This experimental simplicity is important due to the lack of beam viewer in the mid-IR.

\section{MATERIAL AND METHODS}

\section{The Tube Furnace}

The tube furnace used was a Carbolite AGD 12. A schematic view of the furnace is shown in Fig. 1. The geometry and operation are similar to the steady state tube furnace standardized test $[6,7]$. The length of the furnace was $800 \mathrm{~mm}$. A quartz tube with a length of $1700 \mathrm{~mm}$, was fixed in the furnace and protruded from both sides of the furnace. The inner and outer diameters of the quartz tube were 42 and $47 \mathrm{~mm}$, respectively. An $800 \mathrm{~mm}$ long silica boat containing the fuel was delivered into the quartz tube at a speed of $40 \mathrm{~mm} / \mathrm{min}$, in the positive $x$-direction as defined in Fig. 1 . The inner and outer widths of the boat were 36 and $41 \mathrm{~mm}$, respectively. The boat was dragged through the furnace instead of pushed, which is the conventional method of entry of the boat, in order to facilitate the accessibility of the laser optics placed in front of the tube. The constant speed of the boat ensured a continuous fuel feed to the burning point A, see Fig. 1, whose position was normally $x=150-300 \mathrm{~mm}$. In situ measurements inside the furnace were performed and therefore the mixing box prescribed in reference [6] was not used. 


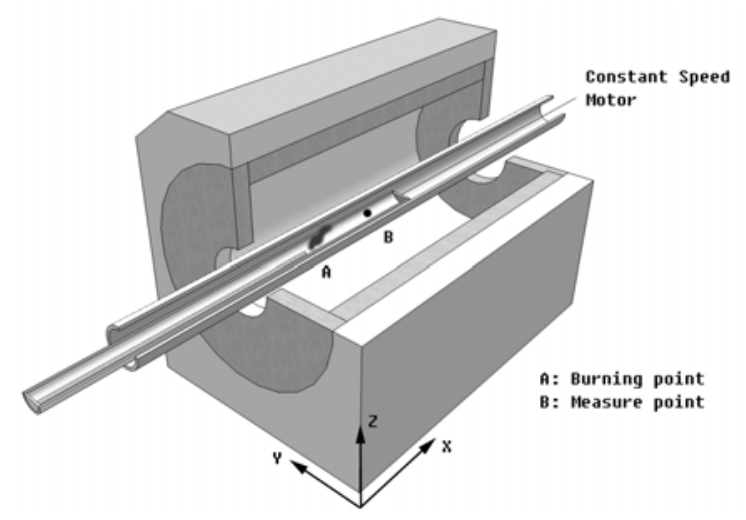

Fig. 1. Schematic view of the cross-section of the tube furnace set-up.

In order to allow the introduction of both an oxidative flow of air and the laser beams into the furnace tube, a Plexiglass box was build, see Fig. 2. The air entered the box through a conduit and the laser beam entered through a $\mathrm{CaF}_{2}$ window. The Plexiglass box was connected to the quartz tube in the furnace via an air tight seal.

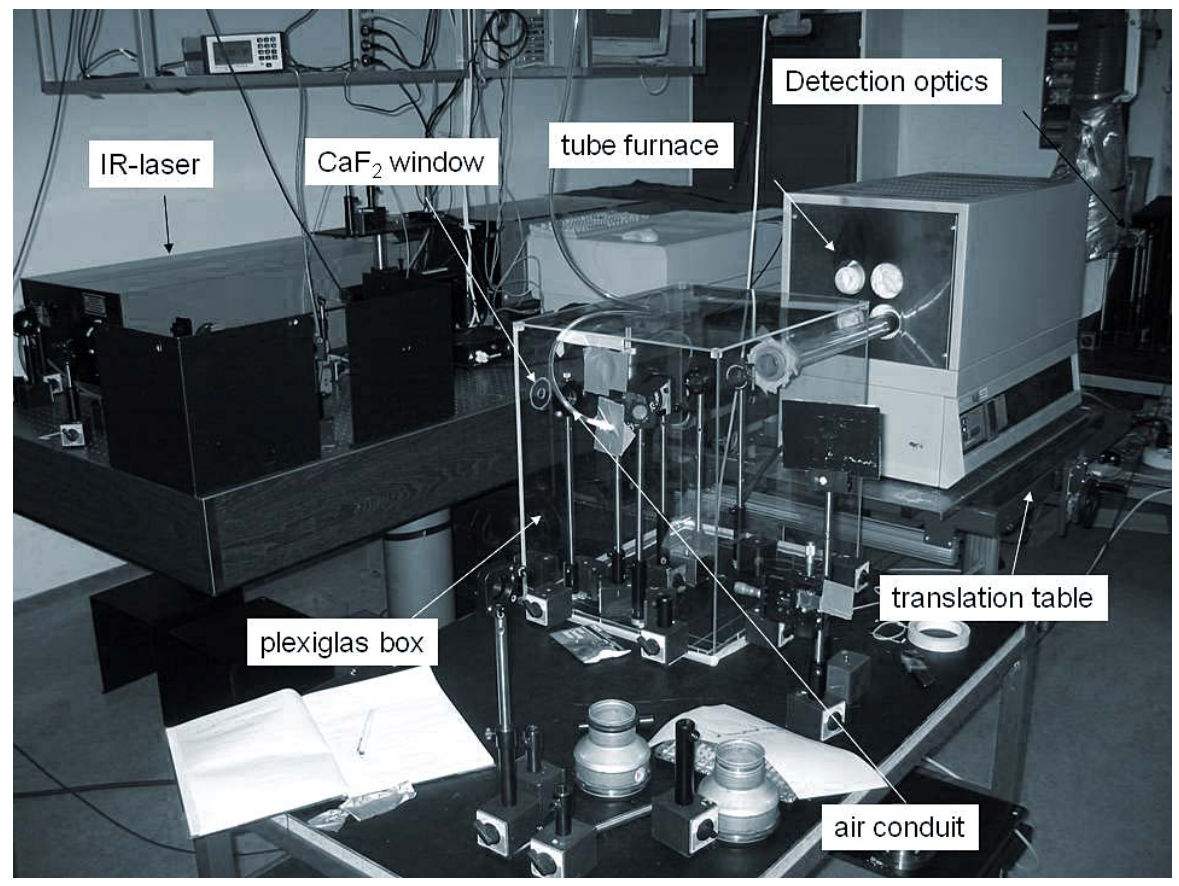

Fig. 2. Photograph of the experimental set-up.

\section{Operating Conditions}

The fuel used in the study was nylon 6,6 pellets (Northern Industrial Plastics). The nylon 6,6 pellets had dimensions of $\sim 2-4 \mathrm{~mm}$ and density $\sim 1140 \mathrm{~kg} / \mathrm{m}^{3}$. The fuel was distributed homogeneously in the boat in order to keep the fuel load constant.

The gas flow through the quartz tube was $10 \mathrm{l} / \mathrm{min}$. In some of the measurements nitrogen was mixed with the air in order to increase the equivalence ratio without creating an exceedingly slow air flow through the furnace. The gas flows were controlled by mass flow controllers (Bronkhorst), and the mixed gas was pumped into the quartz tube in the $x$ direction, see Fig. 1 and Fig. 2. 
The operating conditions for the tests presented in this paper are provided in Table 1. The temperature of the furnace was set using a PID-control for each set temperature, $750{ }^{\circ} \mathrm{C}$ and $910{ }^{\circ} \mathrm{C}$. The actual temperature profile in the middle of the quartz tube was measured using a Type-K thermocouple. The temperature distributions in the furnace are shown in Fig. 3. The equivalence ratios in the experiments were nominally $0.5,1.0$ or 2.0 .

Table 1. Operating conditions

\begin{tabular}{|l|c|c|c|c|c|c|}
\hline Figure & $\begin{array}{c}\boldsymbol{X} \\
(\mathbf{m m})\end{array}$ & $\begin{array}{c}\boldsymbol{T}_{\text {set }} \\
\left({ }^{\mathbf{o}} \mathbf{C}\right)\end{array}$ & $\begin{array}{c}\boldsymbol{T}_{\boldsymbol{x}} \\
\left({ }^{\mathbf{}} \mathbf{C}\right)\end{array}$ & $\begin{array}{c}\text { Nominal } \\
\mathbf{e q .} \text { ratio }\end{array}$ & $\begin{array}{c}\text { Air flow } \\
(\mathbf{l} / \mathbf{m i n})\end{array}$ & $\begin{array}{c}\mathbf{N}_{\mathbf{2}} \text { flow } \\
(\mathbf{l} / \mathbf{m i n})\end{array}$ \\
\hline 6 & 600 & 750 & 698 & 1.0 & 5 & 5 \\
\hline 7 & 600 & 910 & 845 & 1.0 & 5 & 5 \\
\hline 8 & 530 & 750 & 699 & 0.5 & 10 & 0 \\
\hline 9 & 530 & 750 & 699 & 1.0 & 5 & 5 \\
\hline 10 & 600 & 750 & 698 & 1.0 & 5 & 5 \\
\hline
\end{tabular}

${ }^{\mathrm{a}}$ In this study it was simply assumed that the nominal equivalence ratios were equal to the actual equivalence ratios since all nylon 6,6 was combusted.

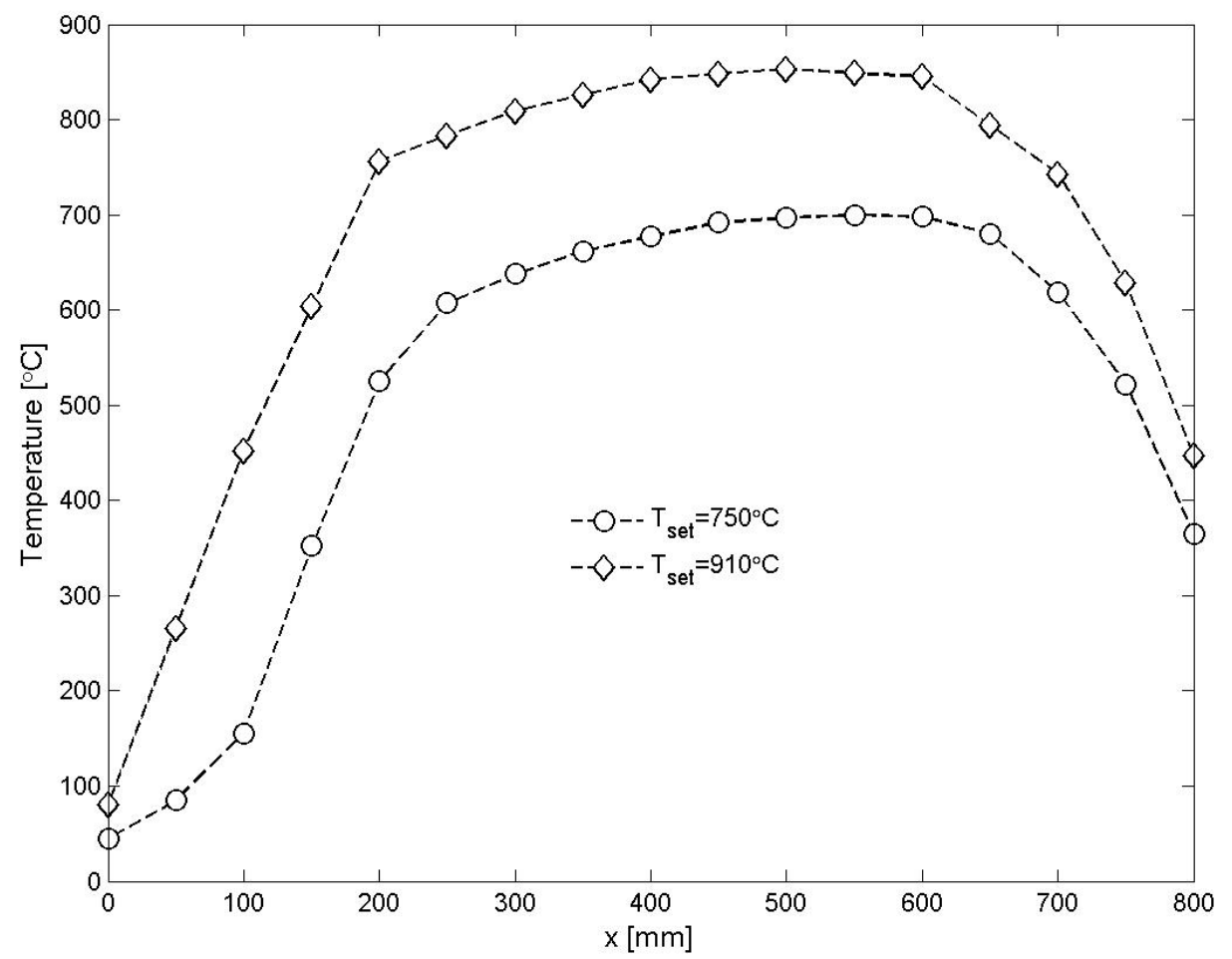

Fig. 3. Temperature distribution in the tube furnace for the set temperatures $750{ }^{\circ} \mathrm{C}$ and $910^{\circ} \mathrm{C}$.

\section{Infrared Polarization Spectroscopy (IRPS)}

A schematic view of the optics set-up used for the experiments is shown in Fig. 4. The pump and probe laser beams were crossed at point B (see Fig. 1) with an angle of $2.2^{\circ}$. The detection volume was approximately $15 \mathrm{~mm} \times 1 \mathrm{~mm} \times 1 \mathrm{~mm}=15 \mathrm{~mm}^{3}(\mathrm{x} \times \mathrm{y} \times \mathrm{z})$. The wavenumber of the IR laser pulses was approximately $3280 \mathrm{~cm}^{-1}$ with a line width of $0.025 \mathrm{~cm}^{-1}$. The laser frequency was constantly scanned over the resonance. The IR beam was traced by a He-Ne laser beam. The IR laser beam was focused by an $f=$ $1000 \mathrm{~mm} \mathrm{CaF}_{2}$ lens, and then approximately $7 \%$ was reflected by a $\mathrm{CaF}_{2}$ plate to be used as the probe beam. The transmitted part of laser beam served as pump beam. A quarter-wave plate was placed in the pump beam to obtain circular polarization. Two $\mathrm{YVO}_{4}$ infrared polarizers were positioned, one before and one after the detection volume, crossed to each other in the probe beam. After passing the probe volume, 
the pump beam was blocked in a beam dump. Part of the probe laser beam reflected from the second polarizer was detected simultaneously in order to record the total absorption along the tube, which gave the absorption spectrum (AS). The PS and AS signals were collected with two independent liquid- $\mathrm{N}_{2}$-cooled InSb detectors, time-integrated and stored in a $1 \mathrm{GHz}$ bandwidth digital oscilloscope. An example of the raw spectra from a measurement in the tube furnace is shown in Fig. 5. The scanning of the laser frequency over the resonance is clearly seen both in the AS and in the PS spectrum. When the scanning reached the end of the range, it was reversed. For a short while, the laser light disappeared which can be seen as the deepest dips in the AS spectrum. Between these dips there are smaller dips which correspond to the resonance, for AS as well as for PS.

The furnace was fixed on a table which could be moved in three directions (x, y, z). This enabled measurements at almost any point in the tube. A photograph of the laboratory is shown in Fig. 2.

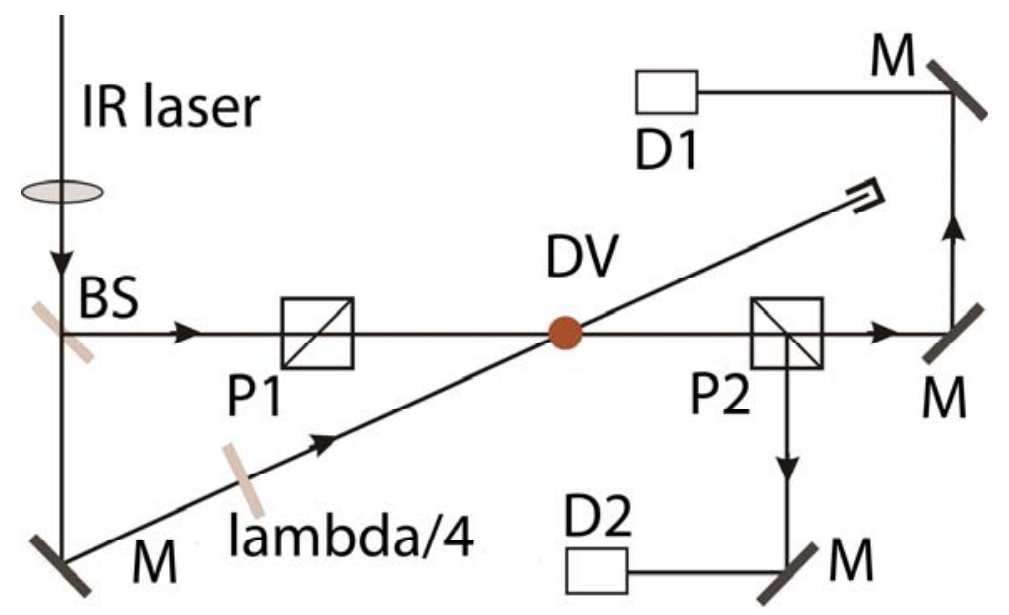

Fig. 4. Schematic view of the optics set-up. IR laser is the laser beam from the laser system, BS beam splitter, P1 and P2 polarizers, D1 detector for the IRPS-signal, D2 detector for the absorption measurements, DV detection volume (inside the furnace, corresponding to point B in Fig. 1), M mirrors.

In order to obtain quantitative information from the PS signal, the integrated intensities of the PS signal was calibrated by HCN sample gases with known concentration. In the experiments, the commercial $\mathrm{HCN} / \mathrm{N}_{2}$ (206 ppm HCN by volume) bottle gas was diluted further by $\mathrm{N}_{2}$ and used for calibrations. The measurement of calibration gases was performed at room temperature and atmospheric pressure. Thanks to the translation table, the furnace could be removed during calibrations. After calibration, the table was repositioned at its original position. This enabled calibrations without re-alignment of the system before measurements could be performed in the furnace.

A detailed description of the theory for polarization spectroscopy can be found in Ref. [26]. Only a short account is given here concerning how quantitative information can be obtained from a spectrum such as the one shown in Fig. 5. 


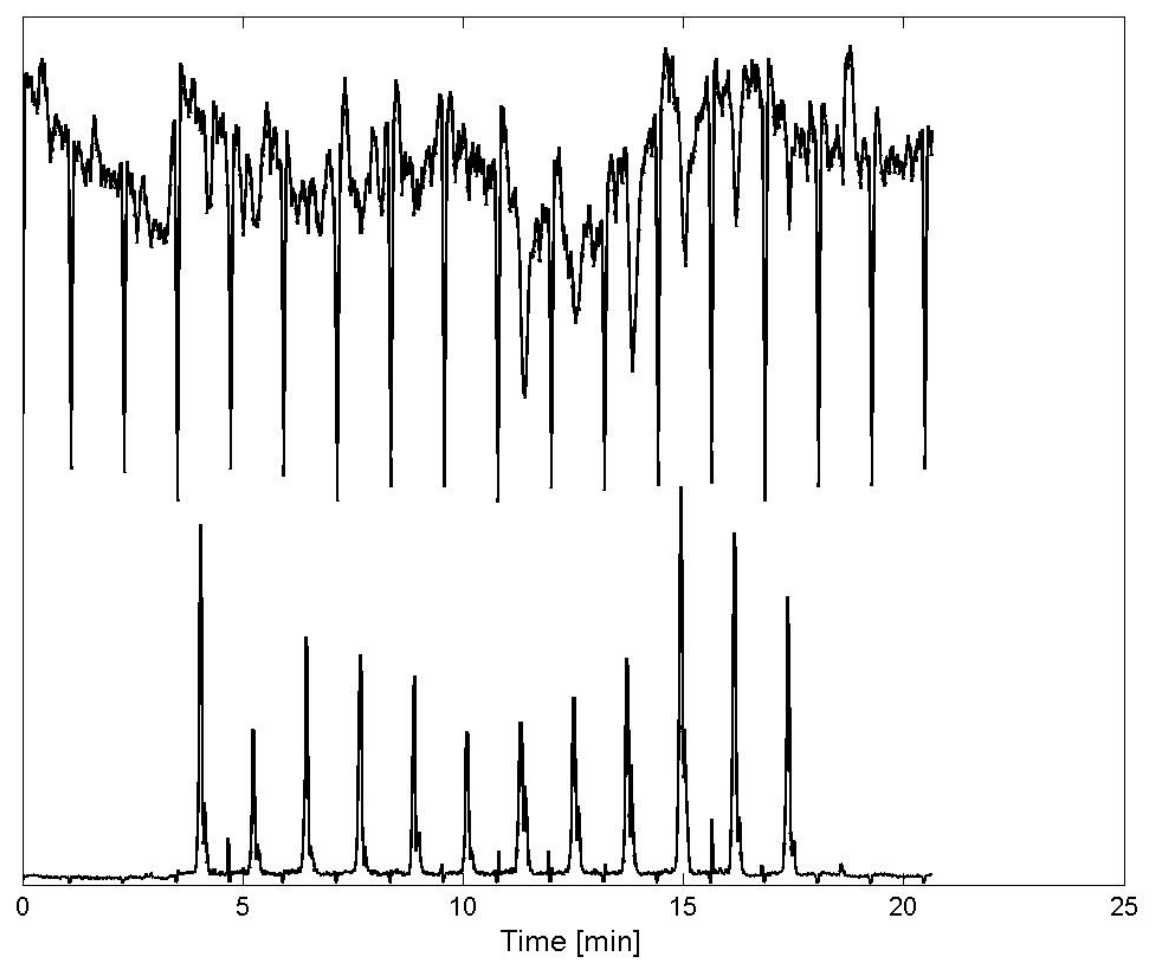

Fig. 5. Raw spectra for the results presented in Fig. 9. The upper spectrum is the absorption signal and lower spectrum is the polarisation signal. The unit on the y-axis is arbitrary.

Experimentally, PS line-integrated signals under saturated conditions [26] can be expressed by the empirical equation [16]:

$I^{p s}=(1-a) \cdot \alpha \cdot g \cdot c \cdot I^{\text {laser }} \cdot \zeta_{J J^{\prime}}^{2} \cdot N_{0}^{2} \cdot \sigma^{2}$

where the parameter $a$ accounts for the non-resonant absorption, $\alpha$ is a factor corresponding the signal collection efficiency; $I^{P S}$ is the IRPS line-integrated signal intensity; $I^{\text {laser }}$ is the probe laser pulse energy; $g$ is a correction parameter accounting for the spectral overlap between the laser profile and the absorption profile of the molecular line; $c$ is a parameter that corrects for the collision effect under different conditions (temperatures, pressures and buffer gases); $\zeta_{J J}$ is a geometry factor of the probed transition, which depends on the pumping geometry and angular momentum of both the upper and lower states; $N_{0}$ is the molecular number density of the probed species; and $\sigma$ is the absorption cross section of the probed transition. Probing the same transition using the same experimental set-up in a calibration gas and in the furnace, the parameters $\zeta_{J J^{\prime}}$ and $I^{\text {laser }}$ were assumed to be the same.

Using the mole fraction $f\left(f=N_{0} / n\right.$, where $n$ is the gas molecule number density following the ideal gas law) and a simple derivation, the mole fraction $f$ of the investigated species in the furnace can be expressed as

$f_{2}=f_{1} \cdot \frac{T_{2}}{T_{1}} \cdot \frac{\sigma_{1}}{\sigma_{2}} \cdot\left(\frac{1}{1-a_{2}}\right)^{\frac{1}{2}} \cdot\left(\frac{\alpha_{1}}{\alpha_{2}}\right)^{\frac{1}{2}} \cdot\left(\frac{g_{1}}{g_{2}}\right)^{\frac{1}{2}} \cdot\left(\frac{c_{1}}{c_{2}}\right)^{\frac{1}{2}} \cdot\left(\frac{I_{2}^{P S}}{I_{1}^{P S}}\right)^{\frac{1}{2}}$

where $f$ and $T$ are the mole fraction of the detected species and the temperature of the gaseous system at the detection point, respectively, while the subscripts 1,2 denote the calibration gas and in the furnace, respectively. The cross sections $\sigma$ of the investigated transition at different temperatures, such as room 
temperature and furnace temperature, were extracted from the HITRAN database [27] and only their relative values are needed here. It should be noted that $\sigma$ both in the HITRAN database and in Eq. (2) are Boltzmann averaged values according to the considered temperature. A value of $(1 / 0.27)$ for $\alpha_{1} / \alpha_{2}$ was adopted in the calibration, based on the measurements. This is a result of the fact that the PS signals were weakened due to thermal gradient effects in the furnace including beam expansion and beam steering. The value was found to be relatively constant for the operating conditions considered here. The ratios $g_{1} / g_{2}$ and $c_{1} / c_{2}$ were given the values of $1 / 1.5$ and $1 / 5$. These values are explained in detail in Ref. [28]. The parameter $a$ accounts for the signal loss due to the absorption of smoke along the furnace tube and the magnitude of $a$ is calculated based on the AS recorded simultaneously. In the calibration gas there was no non-resonant absorption so $\left(1-a_{1}\right)=1$.

\section{RESULTS}

The operating conditions for the experiments presented in Fig. 6 to Fig. 11 are tabulated in Table 1. It should be noted that the AS-measurements are not highly sensitive in contrast to the PS-measurements which are highly sensitive. The non-resonant absorption spectra mainly correspond to interaction of the laser light with soot particles. The resonant absorption spectra correspond to absorption by HCN.

The dependence of HCN levels on temperature is illustrated in Fig. 6 and Fig. 7.
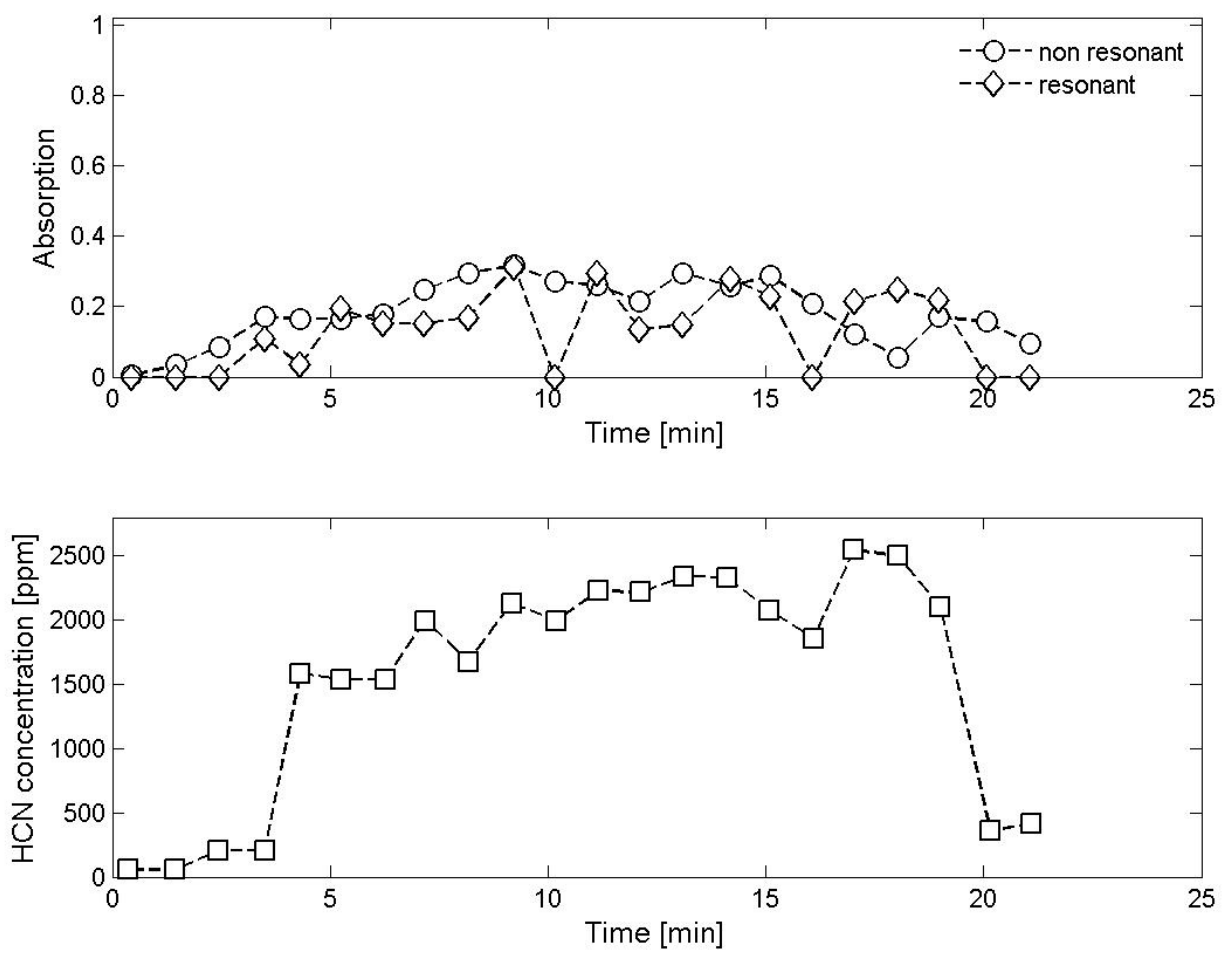

Fig. 6. Measurement at $x=600 \mathrm{~mm}, \varnothing=1$ and the temperature of the furnace was nominally $750{ }^{\circ} \mathrm{C}$. 

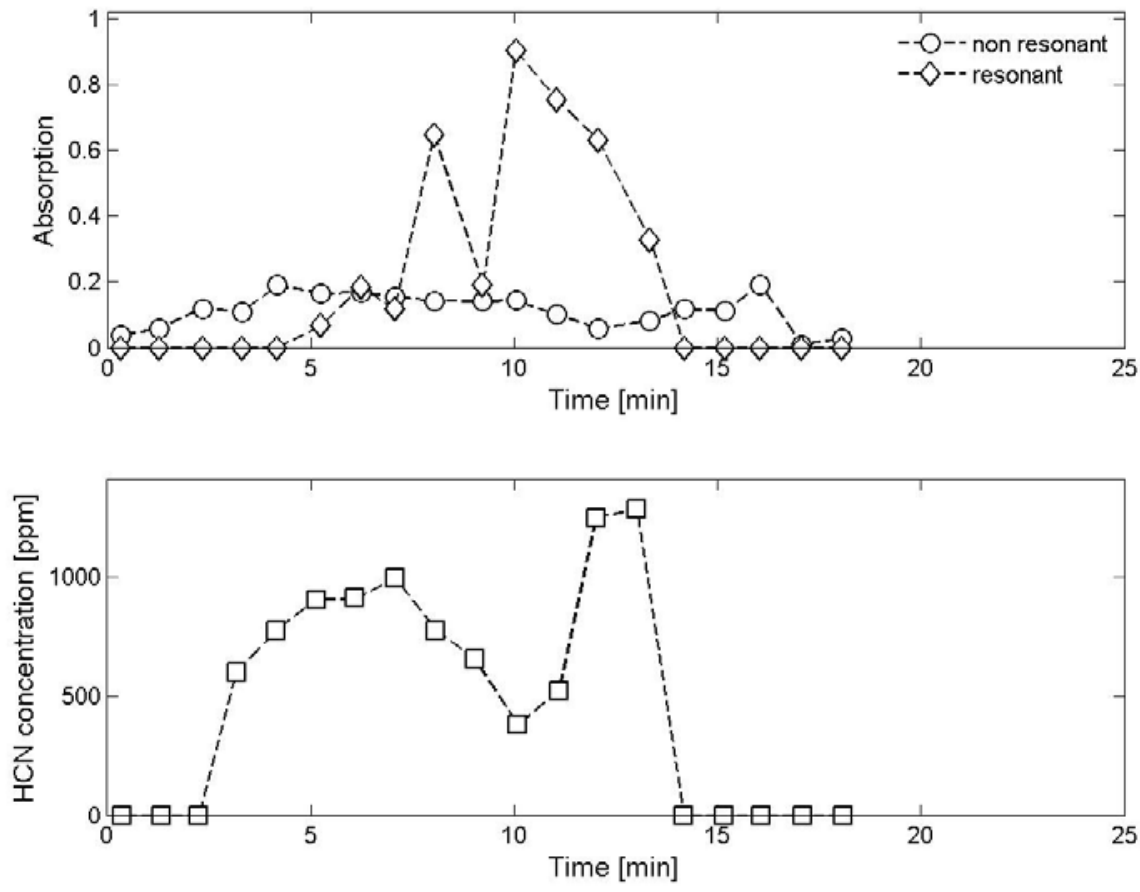

Fig. 7. Measurement at $x=600 \mathrm{~mm}, \varnothing=1$ and the temperature of the furnace was nominally $910{ }^{\circ} \mathrm{C}$.

When Fig. 6 is compared to Fig. 7 it is seen that the total integrated absorption by HCN, i.e. the resonant absorption, increases with increasing temperature. On the other hand the measured concentration of HCN at $x=600 \mathrm{~mm}$, i.e., far downstream of the fire plume, decreased with increasing temperature. An explanation for this is that more HCN is produced in the pyrolysis and/or combustion process when the temperature increases but at the same time the destruction of HCN in the hot gases increases with temperature [1,2].

Figure 8 and Fig. 9 show the dependence of HCN levels on the equivalence ratio.
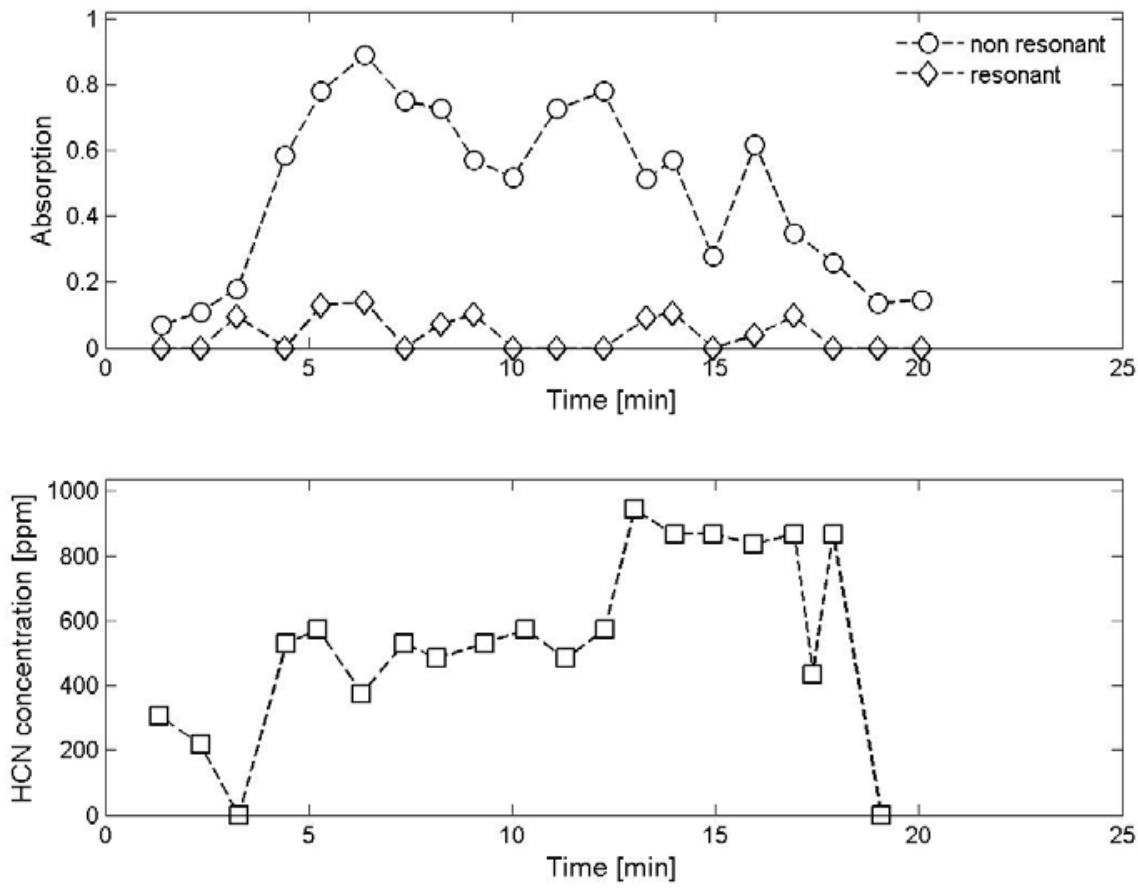

Fig. 8. Measurement at $x=530 \mathrm{~mm}, \varnothing=0.5$ and the temperature of the furnace was nominally $750{ }^{\circ} \mathrm{C}$. 

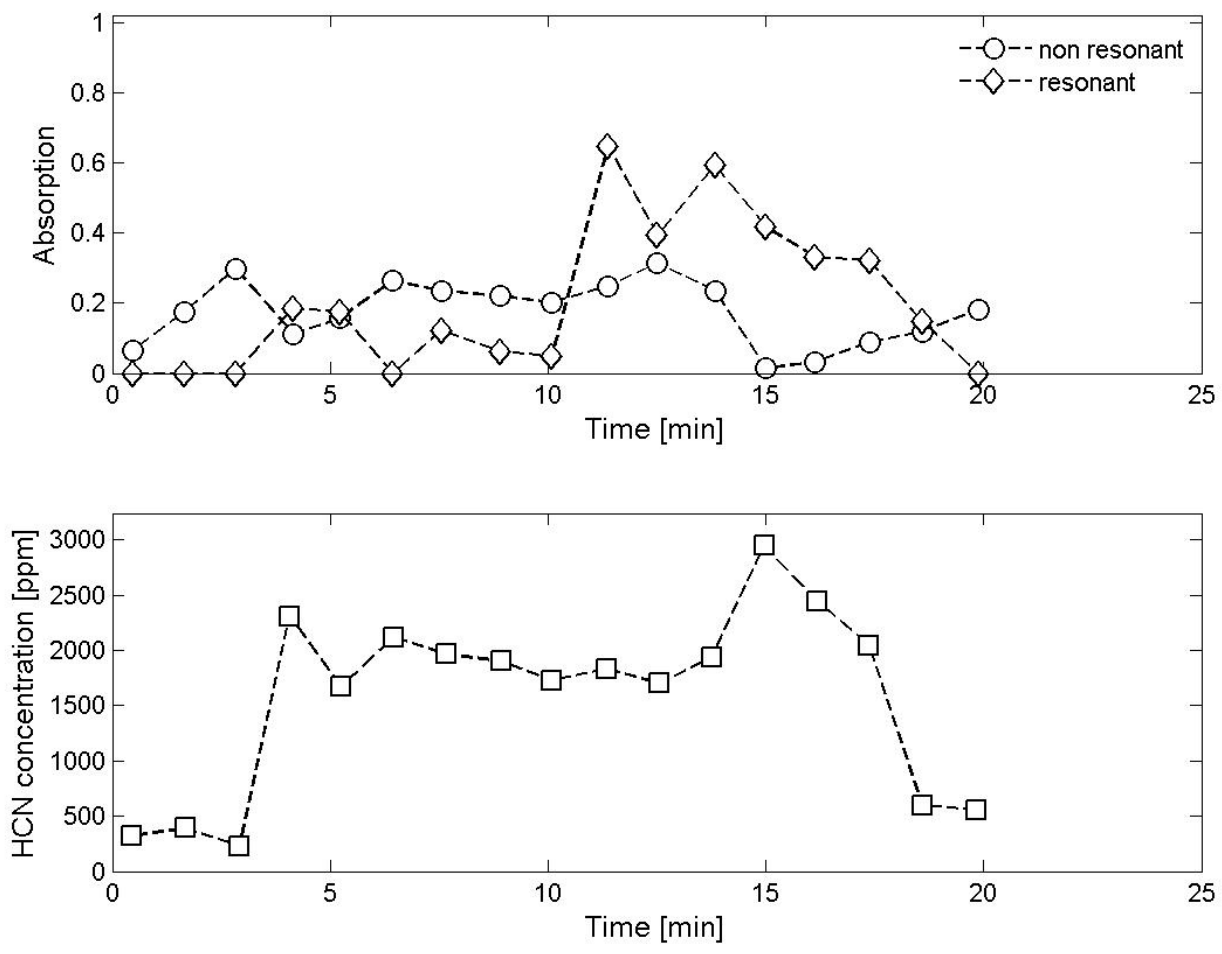

Fig. 9. Measurement at $x=530 \mathrm{~mm}, \varnothing=1$ and the temperature of the furnace was nominally $750{ }^{\circ} \mathrm{C}$.

Comparing Fig. 8 with Fig. 9 it is observed that the amount of HCN, both totally integrated (resonant absorption) as well as that measured at $x=530 \mathrm{~mm}$ with PS, increases significantly when the equivalence ratio increases from 0.5 to 1 . The increase in HCN with increasing equivalence ratio can be due both to an increased production in the pyrolysis and combustion zone but also to a decreased destruction in the hot gases.

The destruction of HCN in the hot gases [1,2] is further supported by the concentration curves in Fig. 10 $(x=600 \mathrm{~mm})$ and Fig. $11(x=700 \mathrm{~mm})$ which show that the HCN concentration decreases slightly as the measurement point is moved downstream in the furnace. 

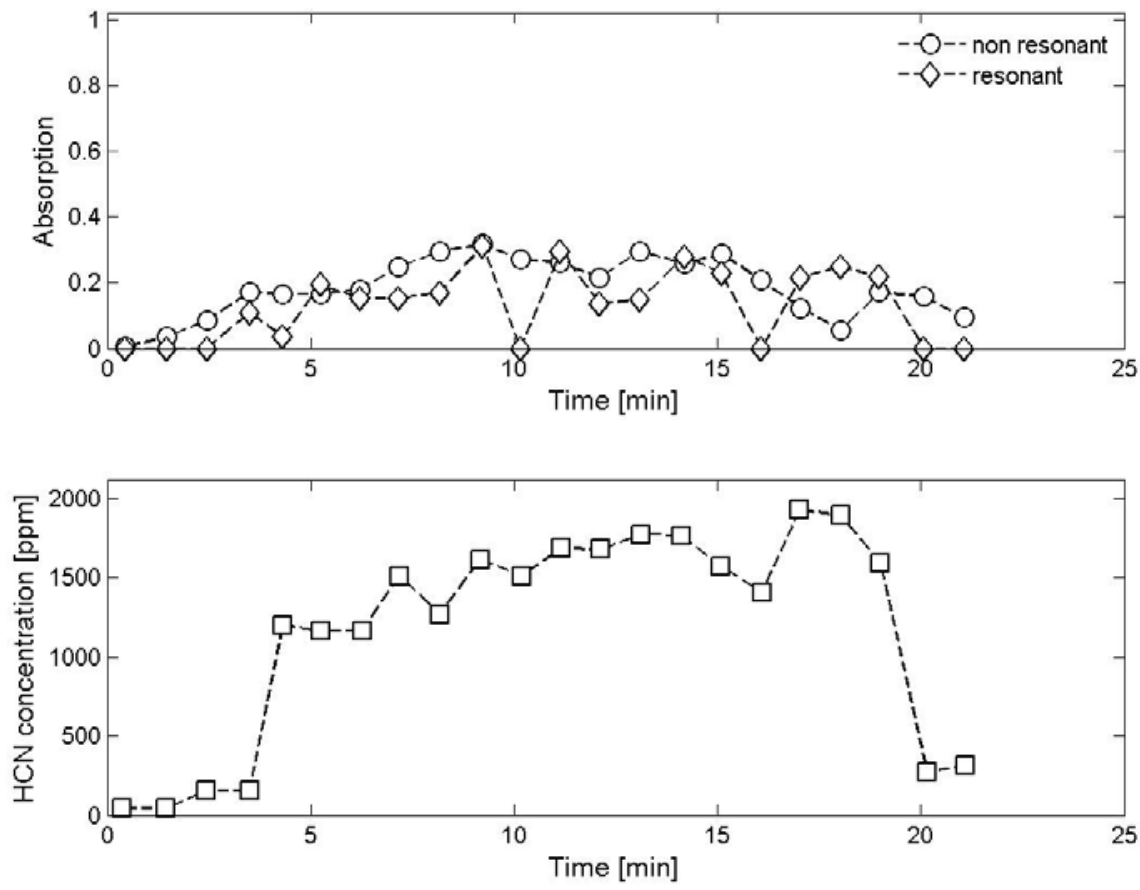

Fig. 10. Measurement at $x=600 \mathrm{~mm}, \varnothing=1$ and the temperature of the furnace was nominally $750{ }^{\circ} \mathrm{C}$.
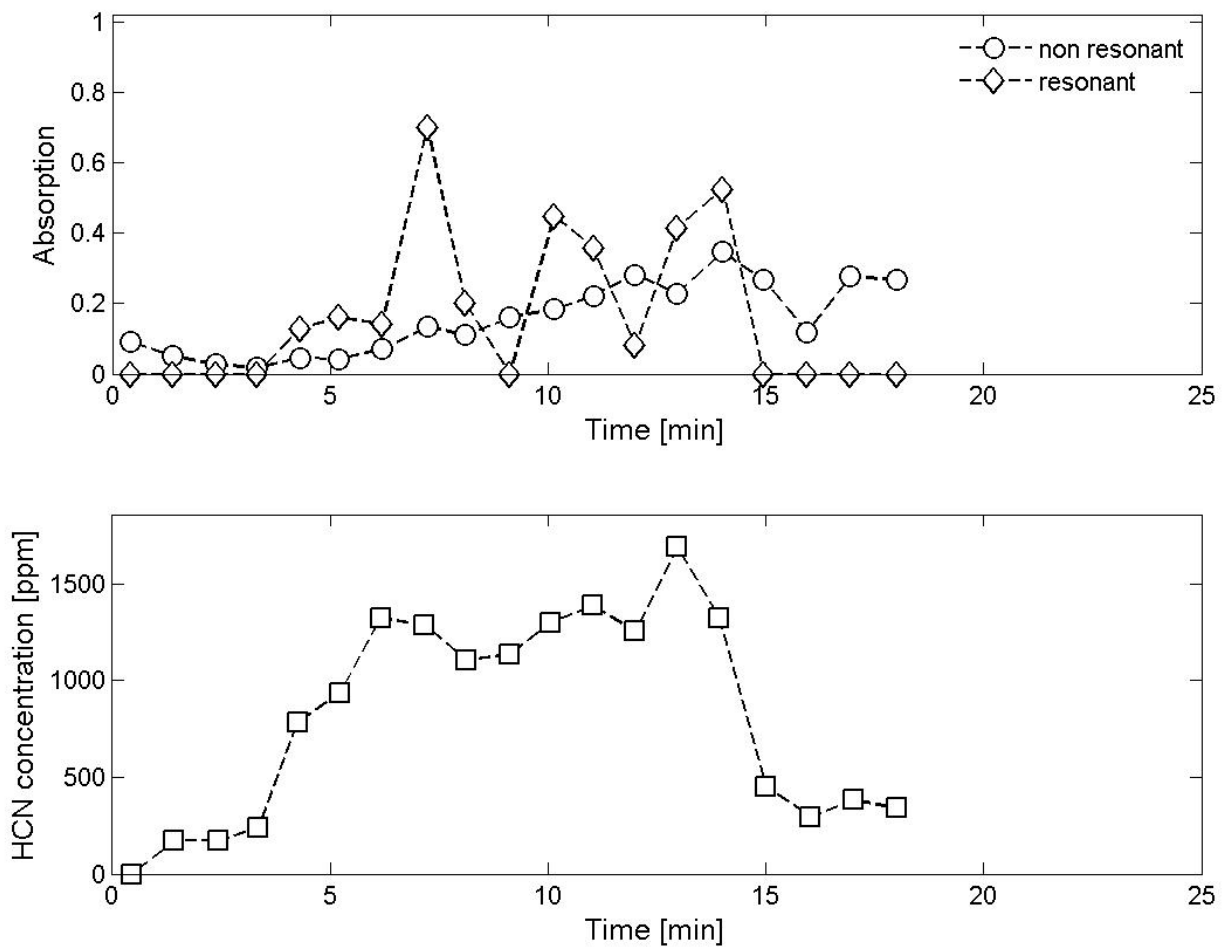

Fig. 11. Measurement at $x=700 \mathrm{~mm}, \varnothing=1$ and the temperature of the furnace was nominally $750{ }^{\circ} \mathrm{C}$.

A general conclusion from the measurements is that the variation in time is relatively high, both for the pointwise IRPS-measurements and for the integrated resonant absorption measurements. This phenomenon is difficult to detect when measurements are conducted in the mixing box, which is traditionally the case in the tube furnace [6]. 
Finally, Fig. 12 shows the variation in time-averaged HCN concentrations with position in the tube furnace for three different operating conditions. It is seen that, for a set temperature of $750{ }^{\circ} \mathrm{C}$, the maximum $\mathrm{HCN}$ concentration measured is obtained for $\varnothing=1$, as compared to $\varnothing=2$. However, the decrease in concentration with distance along the furnace is also more important for $\varnothing=1$ than for the under-ventilated condition where $\varnothing=2$. It is seen that at $x=700 \mathrm{~mm}$, i.e. near the furnace exit, the HCN concentration is higher for $\varnothing=2$.

Another interesting observation is that for the same equivalence ratio, $\varnothing=2$, but for a different set temperatures $750{ }^{\circ} \mathrm{C}$ and $910{ }^{\circ} \mathrm{C}$, the highest peak HCN concentration occurs for the highest furnace temperature. At the same time the decrease in HCN concentration with distance along the furnace is most important for the highest temperature. Again the curves cross and the operating conditions with highest peak HCN concentration inside the furnace end up with a lower HCN concentration near the tunnel exit. These are all observations which support the existing models for HCN production and consumption [2].

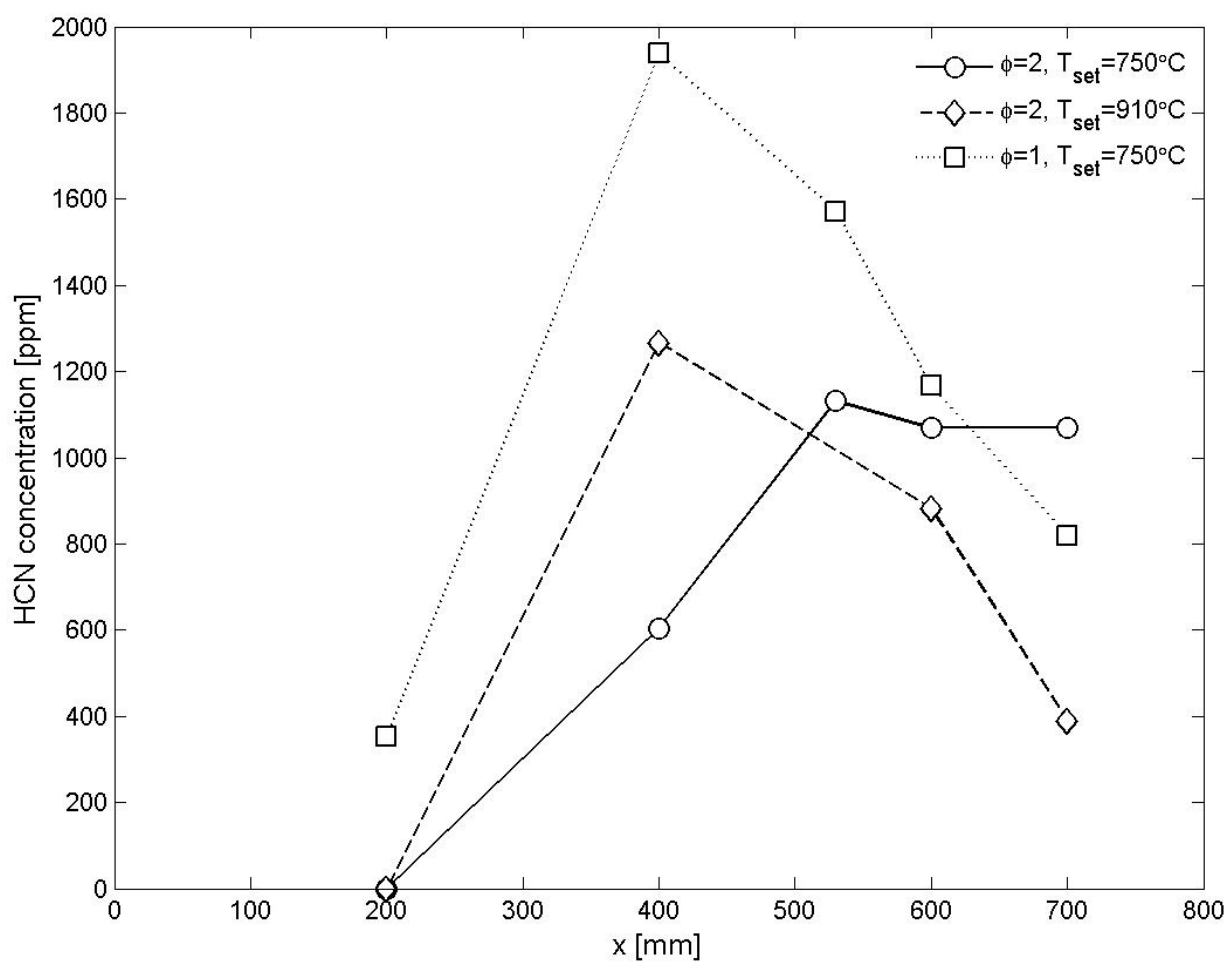

Fig. 12. Time averaged HCN concentration as a function of position in the furnace.

\section{CONCLUSIONS}

IRPS has been successfully applied to spatially resolved in situ measurements inside the steady state tube furnace. In addition line-of-sight absorption measurements of HCN were performed. It was found that the concentration, at the measurement point, varied substantially with time.

It was found that the highest concentration of HCN in the furnace occurred close to the fire plumes. The concentrations then decreased as the smoke and air mixture was transported towards the furnace exit. The highest peak HCN concentrations were measured when the temperature in the furnace was high and ventilation good. At the same time the consumption of HCN during transport with the effluents was most effective for these conditions. This lead to the result that the actual HCN concentrations leaving the furnace were lower for these conditions despite the relatively high HCN concentrations initially. These are in qualitative agreements with existing models for HCN chemistry.

Finally the choice to study the combustion chemistry inside the tube furnace might seem somewhat exotic. However, the successful measurements of HCN inside a smoke laden, narrow and long tube furnace in a spatially and temporally resolved manner demonstrates that IRPS is a powerful tool for toxicity investigation in fire science. In addition to the investigations of fire chemistry in the steady state tube 
furnace we believe that the method is also suitable fire tests such as, for example, the smoke density chamber ISO 5659 [29] and full scale tests in the room-corner test ISO 9705 [30].

\section{ACKNOWLEDGEMENTS}

This work was financed by SSF (Swedish Foundation for Strategic Research), Swedish Energy Agency through CECOST (Centre for Combustion Science and Technology), VR (Swedish Research Council), and Brandforsk Project 303-071.

\section{REFERENCES}

[1] Tuovinen, H., Blomqvist, P., "Effect of residence time on fire gases - experiments and simulations," SP Report 2007:71, Borås, 2007.

[2] Dagaut, P., Glarborg, P., and Alzueta, M., (2008) The oxidation of hydrogen cyanide and related chemistry, Progress in Energy and Combustion Science 34(1): 1-46, http://dx.doi.org/10.1016/j.pecs.2007.02.004

[3] Glarborg, P. and Miller, J., (1994) Mechanism and modeling of hydrogen cyanide oxidation in a flow reactor, Combustion and Flame 99(3-4): 475-483, http://dx.doi.org/10.1016/0010$\underline{\text { 2180(94)90039-6 }}$

[4] Purser, D.A., "Asphyxiant components of fire effluents," Fire toxicity, Woodhead Publishing Limited, Cambridge, 2010.

[5] Fenimore, C.P., "Formation of nitric oxide in premixed hydrocarbon flames," Symposium (International) on Combustion, Volume 13(1):373-380, Combustion Institute, 1971.

[6] "ISO/TS 19700 Controlled equivalence ratio method for the determination of hazardous components of fire effluents," International Organization for Standardization, Geneva, 2007.

[7] Purser, D. and Purser, J., (2009) HCN yields and fate of fuel nitrogen for materials under different combustion conditions in the ISO 19700 tube furnace and large -scale fires, Fire Safety Science 9: 1117-1128, http://dx.doi.org/10.3801/IAFSS.FSS.9-1117

[8] Warnatz, J., Maas, U., Dibble, R.W., Combustion: Physical and Chemical Fundamentals, Modeling and Simulation, Experiments, Pollutant Formation, Springer Verlag, Berlin, 2001.

[9] Stec, A.A., "Estimation of toxicity during burning of common materials," Fire toxicity, Stec, A.A. and Hull, R., (eds.), Woodhead Publishing Limited, Cambridge, 2010.

[10] Purser, D.A., Fardell, P.J., Rowley, J., Vollam, S., Bridgeman, B., and Ness, E.M., "An improved tube furnace method for the generation and measurement of toxic combustion products under a wide range of fire conditions," Flame Retardants '94 Conference, Interscience Communications LTD, 1994.

[11] Blomqvist, P., Hertzberg, T., and Tuovinen, H., "A small-scale controlled equivalence ratio tube furnace method - experiences of the method and the link to large scale fires," Interflam Conference, Interscience Communications LTD, 2007.

[12] Paul, K., Hull, T., Lebek, K., and Stec, A., (2008) Fire smoke toxicity: The effect of nitrogen oxides, Fire Safety Journal 43(4): 243-251, http://dx.doi.org/10.1016/j.firesaf.2007.10.003

[13] Fardell, P., Guillaume, E., "Sampling and measurement of toxic fire effluent," Fire toxicity, Stec, A.A. and Hull, R., (eds.), Woodhead Publishing Limited, Cambridge, 2010.

[14] Smith, G.P., "Diagnostics for detailed chemical modeling," Applied Combustion Diagnostics, Taylor \& Francis, 2002.

[15] Aldén, M., (1999) Laser spectroscopic techniques for combustion diagnostics, Combustion Science and Technology 149(1): 1-18, http://dx.doi.org/10.1080/00102209908952096

[16] Demtröder, W., Laser Spectroscopy: Basic concepts and instrumentation, Springer Verlag, Berlin, 1996. 
[17] Wieman, C., Hänsch, T.W., (1976) Doppler-Free Laser Polarization Spectroscopy, Physical Review Letters 34: 1170.

[18] Li, Z.S., Rupinski, M., Zetterberg, J., Alwahabi, Z.T., and Aldén, M., (2004) Detection of methane with mid-infrared polarization spectroscopy, Applied Physics B 79(2): 135-138, http://dx.doi.org/10.1007/s00340-004-1475-9

[19] Li, Z.S., Rupinski, M., Zetterberg, J., and Aldén, M., (2005) Mid-infrared PS and LIF detection of $\mathrm{CH}_{4}$ and $\mathrm{C}_{2} \mathrm{H}_{6}$ in cold flows and flames at atmospheric pressure, Proceedings of the Combustion Institute 30(1): 1629-1636, http://dx.doi.org/10.1016/j.proci.2004.08.124

[20] Li, Z.S., Rupinski, M., Zetterberg, J., Alwahabi, Z.T., and Aldén, M., (2005) Mid-infrared polarization spectroscopy of polyatomic molecules: Detection of nascent $\mathrm{CO}_{2}$ and $\mathrm{H}_{2} \mathrm{O}$ in atmospheric pressure flames, Chemical Physics Letters 407(4-6): 243-248, http://dx.doi.org/10.1016/j.cplett.2005.03.102

[21] Roy, S., Lucht, R., and Mcllroy, A., (2002) Mid-infrared polarization spectroscopy of carbon dioxide, Applied Physics B: Lasers and Optics 75(8): 875-882, http://dx.doi.org/10.1007/s00340$\underline{002-1034-1}$

[22] Li, Z.S., Linvin, M., Zetterberg, J., Kiefer, J., and Aldén, M., (2007) Mid-infrared polarization spectroscopy of $\mathrm{C}_{2} \mathrm{H}_{2}$ : Non-intrusive spatial-resolved measurements of polyatomic hydrocarbon molecules for combustion diagnostics, Proceedings of the Combustion Institute 31(1): 817-824, http://dx.doi.org/10.1016/j.proci.2006.08.054

[23] Li, Z., Hu, C., Zetterberg, J., Linvin, M., and Aldén, M., (2007) Mid-infrared polarization spectroscopy of $\mathrm{OH}$ and hot water in low pressure lean premixed flames, The Journal of Chemical Physics 127(8): 084310, http://dx.doi.org/10.1063/1.2759216

[24] Li, Z., Sun, Z., Li, B., Aldén, M., and Försth, M., (2008) Spatially resolved trace detection of HCl in flames with mid-infrared polarization spectroscopy, Optics Letters 33(16): 1836, http://dx.doi.org/10.1364/OL.33.001836

[25] Sun, Z., Försth, M., Li, Z., Li, B., and Aldén, M., (2010) Mid-infrared polarization spectroscopy: A tool for in situ measurements of toxic gases in smoke-laden environments, Fire and Materials, in press, http://dx.doi.org/10.1002/fam.1071

[26] Eckbreth, A.C., Laser Diagnostics for Combustion Temperature and Species, Gordon and Breach Science Publishers SA, Amsterdam, 1996.

[27] Rothman, L., Gordon, I., Barbe, A., Benner, D., Bernath, P., Birk, M., Boudon, V., Brown, L., Campargue, A., and Champion, J., (2009) The HITRAN 2008 molecular spectroscopic database, Journal of Quantitative Spectroscopy and Radiative Transfer 110(9-10): 533-572, http://dx.doi.org/10.1016/j.jqsrt.2009.02.013.22 23

[28] Sun, Z., Li, Z., Li, B., Alwahabi, Z., and Aldén, M., (2010) Quantitative $\mathrm{C}_{2} \mathrm{H}_{2}$ measurements in sooty flames using mid-infrared polarization spectroscopy, Applied Physics B 101(1-2): 423-432, http://dx.doi.org/10.1007/s00340-010-4057-z

[29] "ISO 5659:2006 Plastics - Smoke generation," International Organization for Standardization, Geneva, 2006.

[30] "ISO 9705 Fire tests - Full-scale room test for surface products," International Organization for Standardization, Geneva, 1993. 